\title{
Classification of a Satellite Rural Image based on Fractal Dimension using Box Counting Method
}

\author{
DR.Ambika \\ BMS College of Engineering \\ Bull Temple Road \\ Bangalore - 560019
}

\author{
AG Ananth \\ RV College of Engineering \\ Kengeri \\ Bangalore - 560059
}

\begin{abstract}
Fractal geometry has sparked considerable interest for analyzing remote sensing imageries. Fractal models have been used in several image processing and pattern recognition applications such as texture analysis and classification. Applications of fractal geometry in remote sensing rely heavily on estimation of the fractal dimension(D). When land areas are clustered into groups of similar land cover, one of the most important things is to extract the key features of a given image. The present paper uses box counting method for determining the fractal dimension $\mathrm{D}$ for the classification of remote sensing imageries and identifying the various features present in the imageries. The features present in a typical remotely sensed Rural image such as Water bodies, Dry land, Vegetation, Forest, Rocky regions and Housing etc have been studied using the fractal dimensions. The studies show that the box counting method provide a very clear distinction between the fractal dimensions determined for the different features present in the satellite Rural imageries.
\end{abstract}

\section{Keywords}

Fractal Dimension, Remote Sensing, Box

\section{INTRODUCTION}

Remote sensing includes two kinds of information: spectral and spatial. Currently, remote sensing classification is mainly dependent on spectral information with spatial structure information rarely used. Therefore, there is a loss in the remote sensing information, because the spectral and spatial information should be equally important in remote sensing classification ${ }^{(1)}$. Fractal geometry introduced by Mandelbrot ${ }^{(2)}$ describes the irregular or fragmented shapes of natural features as well as other complex objects that the traditional Euclidean geometry fails to analyze.

Most of the nature phenomena exhibit fractal characteristics ${ }^{(3,10)}$ hence; fractal modeling of natural surfaces can play an important role in remote sensing for interpretation of surface physical phenomena, target detection and classification. Many natural surfaces exhibit a fractal behavior which can be synthetically described by an intuitive parameter, the fractal dimension, which is related to the concept of surface roughness (4). Therefore, applications of fractal techniques to image analysis rely heavily on the estimation of fractal dimension, denoted as D. There are many computing methods for fractal dimension. The common important parameters are similar dimension, Hausdorff-Besicovitch dimension ${ }^{(5)}$, information dimension and box dimension etc.
Fractal self similarity character, which means that magnifying local component structure is similar to the whole object structure in some ways ${ }^{(6)}$ is the essential nature of all fractal objects.

The present paper uses box counting method for the determination of fractal dimension in low resolution, satellite rural imagery. The paper introduces the steps and techniques of this method in detail and compares the fractal dimension parameter for different features seen in remote sensing for classification.

\section{THE PRINCIPLE OF BOX COUNTING METHOD}

The box-counting method can be described as follows. Suppose that $\mathrm{F}$ is a limited fractal figure on a plane. $\mathrm{F}$ is covered with square grids which are built by boxes whose side length is $\delta$ (A grid is namely a box.) The number of boxes intersecting on $F$ is $\mathrm{N}$. If $\mathrm{N}$ is satisfied with the power law $\mathrm{N}(\delta) \propto \delta$-D, then as $\delta \rightarrow 0$, the ratio of logarithm

$$
\mathrm{D}=\frac{\ln N(0)}{\ln \left(\frac{1}{5}\right)}
$$

$\mathrm{D}$ is defined as the box dimension of $\mathrm{F}(7)$. The general process of the box-counting method is as follows. Firstly, the research object is covered by a rectangle. Then the rectangle must be nun-empty. The number of nun-empty grids is 1 . Then the side of the rectangle is divided into two parts, to get 4 grids. The size of the grid is $1 / 2$. These are covered on the object, and the number of the nun-empty grid obtained is $N(1 / 2)$. Then the side of the rectangle is quartered to get 16 grids. The size of the grid is $1 / 22$. These are covered on the object, and the number of the nun-empty grids obtained is $\mathrm{N}(1 / 22)$. This process is continued until the side of the rectangle is divided into $2 \mathrm{n}$ parts to obtain $2 \mathrm{n} \times 2 \mathrm{n}$ grids. These are covered on the object, and the number of the nun-empty grids obtained is $\mathrm{N}(1 / 2 \mathrm{n})$. Here $1 / 2 \mathrm{n}$ is the size of grid $\delta$. Generally, $\mathrm{n}=9$ is chosen to obtain 9 pairs of data of the size and the number of the nun-empty grids. Then observe these 9 pairs of data are observed to check if negative power law $\mathrm{N}(\delta) \propto \delta$-D is satisfied (8). Since the negative power law is equal to the logarithm linear relationship, it may be observed if their distribution in logarithm coordinate system tends to be linear. If it is linear, then the research object is fractal. These points are fitted with least square method. The absolute value of the slope of the fit line is just the fractal dimension of the research object. 


\section{REALIZATION OF THE BOX COUNTING METHOD}

A low resolution satellite rural imagery which includes features such as Water bodies, Dry land, Vegetation, Forest, Rocky regions and Housing etc has been selected for the fractal analysis shown in the figure 1 .

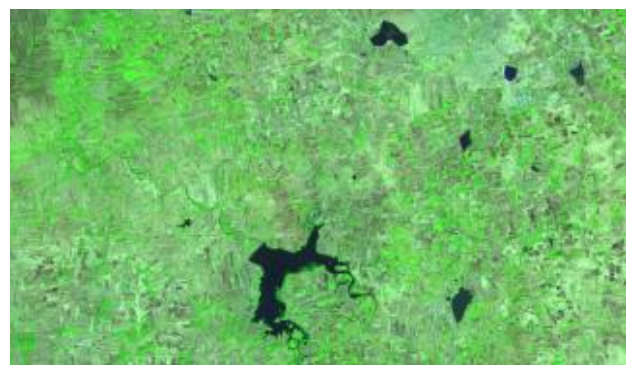

Fig 1: The Research Area

This image data is exported to MATLAB and saved as an 8 - bit gray image (BMP format).

As an example, a $4 \times 4$ gray image is shown in Figure 2.

\begin{tabular}{|c|c|c|c|}
\hline 0 & 1 & 1 & 1 \\
\hline 0 & 0 & 0 & 0 \\
\hline 1 & 0 & 0 & 0 \\
\hline 0 & 1 & 0 & 0 \\
\hline
\end{tabular}

Fig 2: 4 X 4 gray image

This image is divided into 4 sub-images and numbered in turn as A, B, C and D. These are judge for nun-zero sub-matrixes ${ }^{(9)}$. In the 4 sub-images, $\mathrm{A}, \mathrm{B}$ and $\mathrm{C}$ are all nun-zero sub-matrixes, $\mathrm{D}$ is a zero matrix. So, the numbers of the nun-zero sub-matrixes of the $4 \times 4$ image is 3 when it is divided into 4 equal parts. Namely, when the image is covered by a $2 \times 2$ grid, the number of nunempty grids is 3 . In the same way the number of the nun-zero sub-matrixes when the image is divided into 8 equal parts is 5 . On extending this method further, it can be found out that, in order to get the number of the nun-zero sub-matrices, the image is divided into 4 equal-parts to get 4 sub-images. Then each subimage is divided into 4 equal parts and number of the nun-empty sub-matrixes is calculated. The sum of the numbers of the nunempty sub-matrixes of these 4 sub-images is that of the previous image divided into 4 equal parts. Thus the number of the nunzero sub-matrixes is calculated by a recursive algorithm. It is described as follows: to seek the numbers of the nun-zero submatrixes of an image divided into $2^{\mathrm{n}} \times 2^{\mathrm{n}}$ equal parts, this image can first be divided into 4 equal sub-images and the numbers of the nun-zero sub-matrixes of each sub-image divided into $2^{\text {n- }}$ ${ }^{1} \times 2^{\mathrm{n}-1}$ equal parts is calculated. The sum is the numbers of the nun-zero sub-matrixes of the superior image. The calculation of the numbers of the nun-zero sub-matrixes of the sub-image divided into $2^{\mathrm{n}-1} \times 2^{\mathrm{n}-1}$ equal parts is the same as above. The recursive function needs to be executed until $n=1$, namely, when only the number of the nun-zero sub-matrixes divided into 4 equal parts need to be calculated. This recursive algorithm is realized in Matlab and the numbers of the nun-empty grids in 9 sizes in turn are calculated. The logarithm (base e) of the data is obtained and figured out in the coordinate system as shown in Figure 3. The points show a clear linear tendency indicating that the considered rural area has the characteristic of a fractal. Using the least square method these points are fit into a line. The fractal dimension is determined as the slope of the line.

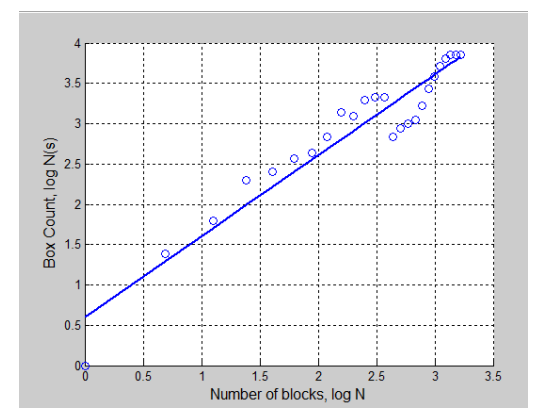

Fig: 3 The $\ln -\ln$ plot

\section{RESULTS AND DISCUSSION}

Based on ground truth data, the different features seen in the satellite Rural image which includes Vegetation, Dry land, Water bodies, Rocky regions, and Housing have been selected for determining the fractal dimensions. The features selected from the satellite Rural image and the corresponding fractal dimensions estimated for the same using techniques describes have been listed in table 1, 2 and 3 . The figure 4 gives the all the important Rural features identified and the estimated fractal dimensions.

It can be seen from the Table 1 that the Rural features such as Housing, Rocky regions and Vegetation across water canals show high fractal dimensions $\sim 1.35$. This indicates clearly the presence of rough surfaces in these features.

Table 1: Fractal Dimensions of Housing, Rocky regions and Vegetation across water canals

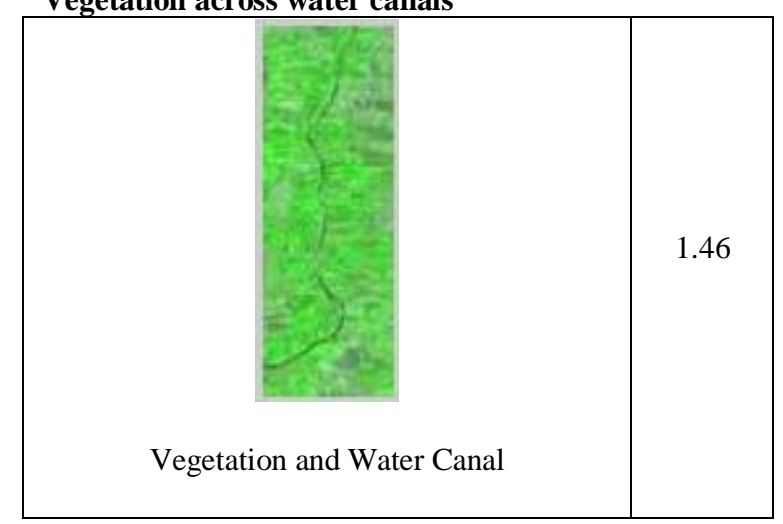




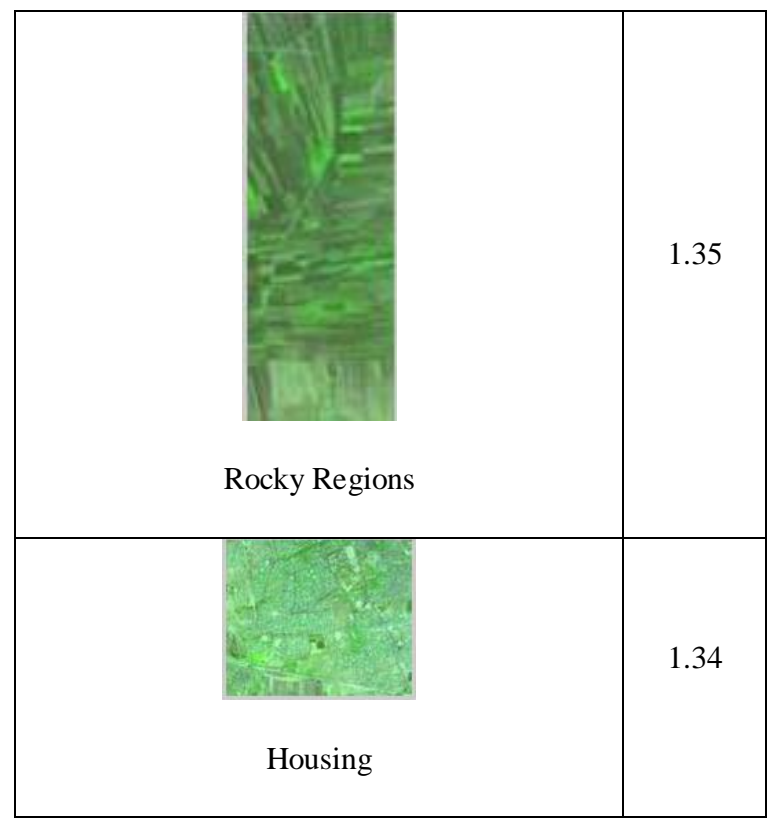

The Table 2 shows that the common Rural features such as Vegetation indicates consistently lower fractal dimensions $\sim 1.2$.

Table 2: Fractal Dimensions of Vegetation

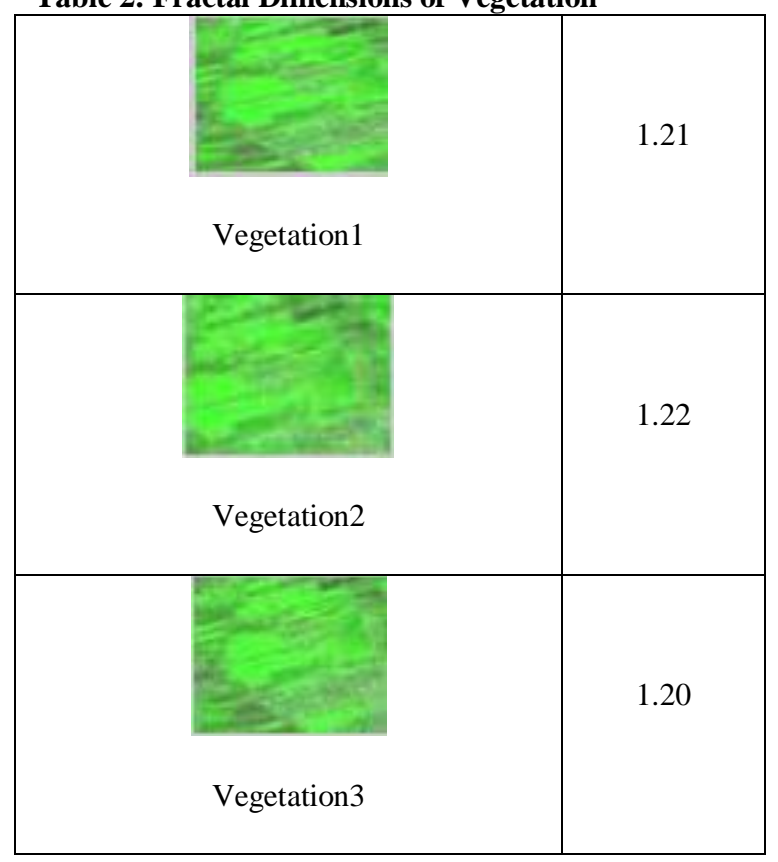

Similarly the Table 3 shows that the abundantly present Rural features such as Water bodies, Dry land etc show a very low fractal dimensions $\sim 1.0$ indicating that they are associated with very smooth surfaces and the absence of any fractals in these objects.
Table 3: Fractal Dimensions of Water bodies and Dry land

\begin{tabular}{|c|c|}
\hline Dry Land 1 & 1.00 \\
\hline Dry Land 2 & \\
\hline Water1 & 0.95 \\
\hline Dry Land 3 & 0.97 \\
\hline Water2 & \\
\hline
\end{tabular}

\section{CONCLUSIONS}

From the results presented above the following conclusions can be drawn.

1. The fractal techniques can be extensively used for identifying the various features present in a satellite Rural image.

2. Box counting method is very effective for deriving the fractal dimensions of the various Rural features such as housing, Vegetation, Rocky regions Dry land and Water bodies etc derived from satellite imageries. 
3. The Rural features like Housing, Rocky regions and Vegetation associated with water canals show higher fractal dimensions ( $\quad 1.35)$ compared to that of Vegetation indicating the presence of rough surfaces.

4. The Rural features such as water bodies and dry land indicate the absence of fractal dimensions $(\sim 1)$ suggesting that the presence of smooth surfaces.

\section{ACKNOWLEDGMENTS}

We are immensely thankful to our family and friends for all the encouragement and support given towards the publication of this manuscript.

\section{REFERENCES}

[1] ZHU Ji, LIN Ziyu, WANG Angsheng, CUI Peng, 2006. An approach to extracting fractal in remote sensing image. WUJNS, Vol.11, No.3,606-610

[2] Mandelbrot, B.B., 1977, Fractals: Form, Chance and Dimension. San Francisco, CA: W.H. Freeman and Company.

[3] W. SUN, G. XU, P. GONG and S. LIANG, 2006. Fractal Analysis of Remotely Sensed Images: A review of methods and applications, Review Article, International Journal of Remote Sensing, Vol. 27, No. 22, 4963-4990.
[4] F. Berizzi, P. Gamba, A. Garzelli, F. Dell' Acqua 2002. Fractal Behavior of Sea SAR ERS1 Images, IEEE transactions.

[5] Qulin TAN, Yun SHAO, Xiangtao FAN.,2002. A Novel Edge Detection Algorithm for Remote Sensing Images based on the self-similarity of Fractal Character, IEEE transactions.

[6] Mandelbrot B. B., 1983. The Fractal Geometry of Nature. New York: Freeman, 20-40.

[7] Ye, J., Chen, B.Z., 2001. The application of the fractal theory in the city research. Urban Planning Forum 4, 38-42.

[8] Jiang, S.G., 2004. Studies on fractal urban form using GIS and remote sensing images of Beijing: theory, method and practice. Paper for the Bachelor's degree of Peking University.

[9] Peng, R.D., Xie, H.P., Ju, Y., 2004. Computation method of Fractal dimension for 2-D digital image. Journal of China University of Mining \& Technology 33: 19-24.

[10] Chen, Y., Chen, L., 1998. The fractal geometry. Beijing: Earthquake Publishing. 